\title{
The Joint Moment Distribution of the Lower Extremity During Tai Chi Gait
}

\author{
Richard Pearlman ${ }^{1}$, John E. Kovaleski ${ }^{2}$, Jonathan Wolfe ${ }^{1}$ and Wei Liu ${ }^{3^{*}}$ \\ ${ }^{1}$ School of Medicine, University of South Alabama, Mobile, Alabama, USA \\ ${ }^{2}$ Department of Health, Physical Education, University of South Alabama, Mobile, Alabama, USA \\ ${ }^{3}$ Edward Via College of Osteopathic Medicine Auburn, Auburn, Alabama, USA \\ *Corresponding author: Wei Liu, Edward Via College of Osteopathic Medicine Auburn, Auburn, Alabama, USA, Tel: 443-909-8221; Fax: 850-939-4152; E-mail: \\ wliu@auburn.vcom.edu
}

Received date: January 26, 2017; Accepted date: February 07, 2017; Published date: February 10, 2017

Copyright: ( $) 2017$ Pearlman R, et al. This is an open-access article distributed under the terms of the Creative Commons Attribution License, which permits unrestricted use, distribution, and reproduction in any medium, provided the original author and source are credited.

\begin{abstract}
Background: As an alternative and complementary practice, Tai Chi is becoming increasingly popular in the United States, especially among the elderly. Although several interventional and qualitative biomechanical studies have been performed, a quantitative approach, such as multi-joints kinetics synergy of ankle, knee and hip, has yet to be performed. To better understand the biomechanics of Tai Chi, the characteristics of the total support moment synergy were studied during Tai Chi gait and compared to normal gait.
\end{abstract}

Methods: Ten healthy, experienced (two years) Tai Chi Chuan practitioners performed normal walking and Tai Chi gait while data was collected using high-speed infrared motion analysis cameras. The joint distributions of the ankle, knee, and hip were calculated by ratio between individual joint moment impulse and total support moment impulse. Using a paired t-test, the joint moment distributions of the lower limb were compared between Tai Chi gait and normal walking.

Results: The total support moment of Tai Chi walking was predominated by the contribution of the knee (ankle: $32.78 \pm 1.25 \%$; knee: $58.68 \pm 1.84 \%$; hip: $8.54 \pm 1.91 \%$ ) whereas the ankle was the primary contributor to support in normal walking (ankle: $67.86 \pm 2.76 \%$; knee: $26.18 \pm 2.69 \%$; hip: $5.96 \pm 1.83 \%$ ). The contributions of all three joints studied differed significantly $(p<0.05)$ between Tai Chi gait and normal gait.

Conclusion: This study demonstrates different kinetics synergy patterns between Tai Chi gait and normal gait. Importantly, Tai Chi gait places a high mechanical demand on the knee joint. Our results provide biomechanical basis of Tai Chi's benefits on increasing knee joint range of motion and muscle strength, also suggest that prescribing Tai Chi as a potential therapy for people with joint disease, it needs a careful consideration of evaluating mechanical response of people with joint disease during Tai Chi exercise due to higher mechanical demand on the knee joint.

Keywords: Tai chi; Total support moment; Gait; Support strategy

\section{Background}

Tai Chi Chuan (TCC) exercise is becoming an increasingly popular complementary and alternative approach for both healthy people and patients with a variety of medical conditions in the United States [1]. In the past two decades, promising evidence suggests that TCC provides multiple beneficial effects that include improved balance, increased postural stability, and decreased fall risk [2]. Increased muscular strength, pain reduction for patients with arthritis, reduction of stress, and enhanced cardiovascular function have been proposed as potential benefits of TCC [3-6].

Although TCC has been shown to have many benefits, the mechanism of TCC at the neuromuscular and musculoskeletal levels is less understood. As a form of physical activity, TCC is a continuous, slow and rhythmic movement, involving dynamic weight shifting strategies between two limbs during loading and unloading. These characteristics of TCC require coordinated control of the ankle, knee, and hip [7]. Quantifying the biomechanical characteristics of TCC will aid in our understanding of the movement strategies used in TCC, provide direct evidence of the neuromuscular and musculoskeletal responses of the human body during TCC, and lead to the development of more effective TCC treatment.

Previous studies on the biomechanical quantifications of TCC mainly focused on essential and basic TCC movements such as Tai Chi gait (TCG) due to the fact that TCC has varieties of forms and styles. We believe TCG to be an ideal movement to study for biomechanical analysis because TCG and walking are both a means of bipedal forward locomotion and each have distinct double support and single stance phases.

Since the same gait analysis terminology has been used to describe TCG, it has been referred to as a specific gait strategy. Past research has examined temporal and spatial measures of TCG, such as stride length; basic kinematic descriptive information, such as range of motion (ankle, knee, and hip joints); and kinetics, such as the ground reaction force (GRF), joint moment, and contact force of the lower extremities $[8,9]$.

This biochemical information gives insight into the movements of TCC by isolating individual joints. The main motor function of walking and TCG is to support the body and prevent lower limb 
collapse, which is described as vertical support. Vertical support of the body during gait requires synergistic muscle moments around the hip, knee, ankle.

The total support moment (TSM) has been used to determine joint distributions of this kinetic synergy effect [10]. The total support moment is a summation of the moments of the individual segments of the limb. Despite considerable variability at individual joints, the total support moment shows decreased variability due to the nature of the limb to act as a system whose individual segments can accommodate changes within that system [10]. As a method of analysis that considers the limb as a single unit comprised of individual parts, the support moment synergy is ideal for studying multi-joint movements. Based on our knowledge, few studies have investigated the multi-joint coordination and distribution of lower extremity joint moments during TCG.

In this paper, we quantify the lower limb joint moment distributions of TSM during TCG compared to walking. We hypothesized that joint moment distributions of TSM during TCG will be different than normal walking $(\mathrm{NW})$.

\section{Methods}

\section{Subjects}

Ten healthy participants ( 5 women, mean age 58 years) were enrolled from local Tai Chi clubs and martial art schools. A minimum of two years of TCC (24 simplified forms derived from Yang Style) experience was required.

Exclusion criteria included significant orthopedic and/or chronic pain conditions, depression, and pulmonary or renal failure. Written informed consent was obtained in compliance with the Institutional Review Board requirements at the University. Subjects were asked to stand quietly in a neutral position to capture calibration trial for five seconds. They were then instructed to perform four NW and TCG trials, respectively.

\section{Data collection}

Seven VICON MX-10 high-speed infrared motion analysis cameras (VICON Technology, CA) were used to record TCG and NW with a sampling rate at $100 \mathrm{~Hz}$. Reflective cluster markers were attached to the bony landmarks at the trunk, pelvis, thigh, shank, and foot (Figure $1)$.

Two force platforms (Advanced Mechanical Technology Inc., Watertwon, MA, USA) embedded in a $3 \mathrm{~m}$ (width) $\times 10 \mathrm{~m}$ (length) walkway were used to record ground reaction forces (GRF) at $1000 \mathrm{~Hz}$ during the stance phase of gait.

The two force plates were synchronized with the VICON cameras so that the coordinates of each marker and GRF were simultaneously collected. Three-dimensional marker data were collected by VICON Nexus Software (VICON, CA) and filtered with a Butterworth lowpass filter (6 Hz cutoff).

Right-handed local segmental and lab coordinate systems were constructed so that the $\mathrm{x}$-axis was directed laterally to the right, the $\mathrm{y}$ axis was directed anteriorly, and the z-axis was directed superiorly. Figure 1 illustrates the experimental set up and the orientation of the global and local coordinate systems.

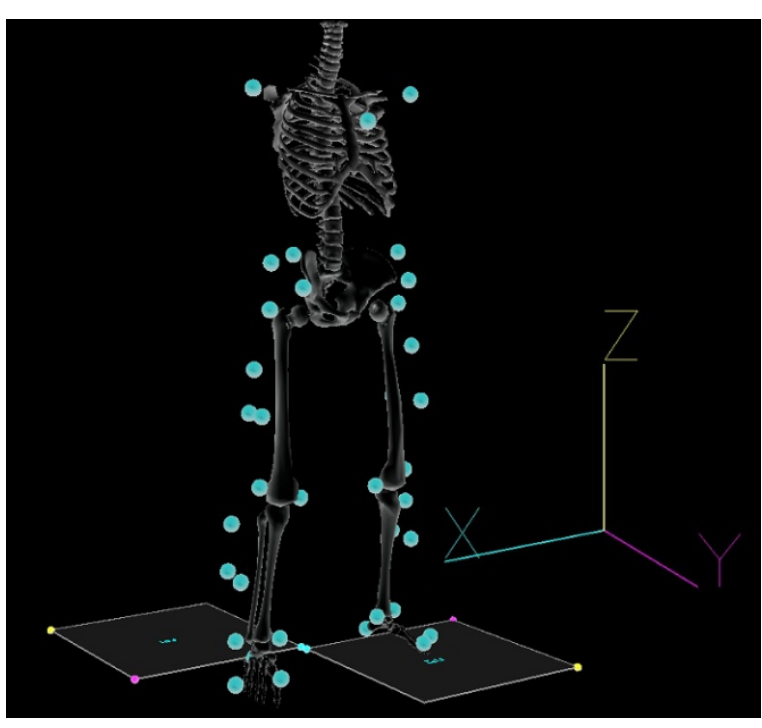

Figure 1: Experimental set-up and marker placement on lower extremity.

\section{Data analysis}

The dependent variables used were distributions of ankle, knee, and hip joint moments of the total support moment. Firstly, 3-D segmental Euler kinematics ( $\mathrm{x}-\mathrm{y}-\mathrm{z}$ sequence) angles were computed based on the rotation matrix between global and local coordinate systems of each segment. Consequently, joint angular displacement, velocity, and segmental angular velocity were derived from 3-D Euler angles [10]. A traditional inverse dynamics approach was used to compute internal joint moment by using the above kinematic and ground reaction force information [10].

The total support moment (TSM) was calculated by summation of the ankle, knee, and hip moments in the sagittal plane [10]. The area under the TSM, called the TSM impulse, was derived by integration of the TSM over the gait cycle.

Finally, the ankle, knee, and hip moment impulses were derived and divided by the TSM impulse. The ratios were then normalized by $100 \%$. A paired t-test was used to compare the ankle, knee, and hip joint distributions to the TSM impulse differences between NW and TCG. The statistical significance level for all comparisons was set at $\mathrm{p}<0.05$.

\section{Results}

\section{Normal gait findings}

Study of ten healthy subjects showed consistent support strategies (Figure 2$)$. The ankle $(67.86 \pm 2.76 \%)$ was found to be the primary contributor to the total support moment.

The knee $(26.18 \pm 2.69 \%)$ participated less than the ankle, and the hip $(5.96 \pm 1.83 \%)$ contributed the least (Figure 3$)$. 


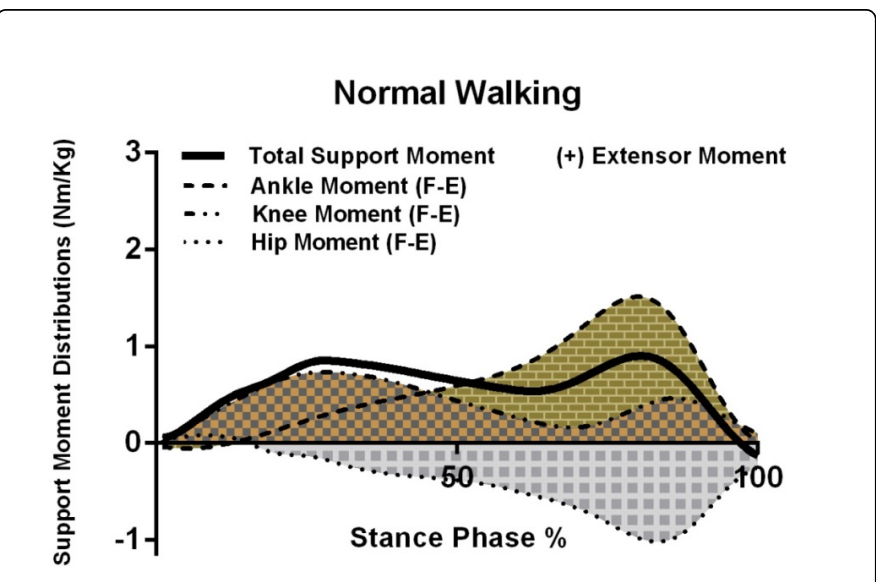

Figure 2: Illustration of time series of Individual joint distributions of total support moment during Normal Walking (NW). Extensor moment $(+)$.

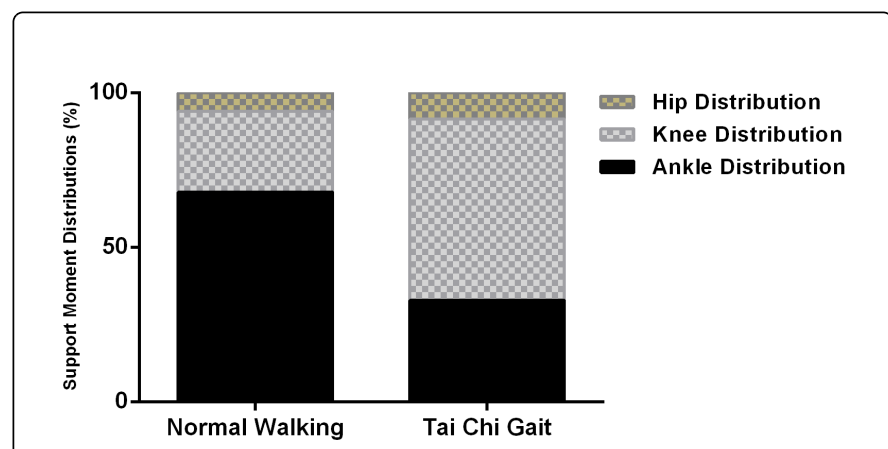

Figure 3: Bar plots of Individual joint distributions of total support moment during normal walking (NW) and Tai Chi Gait (TCG).

\section{Tai Chi Gait}

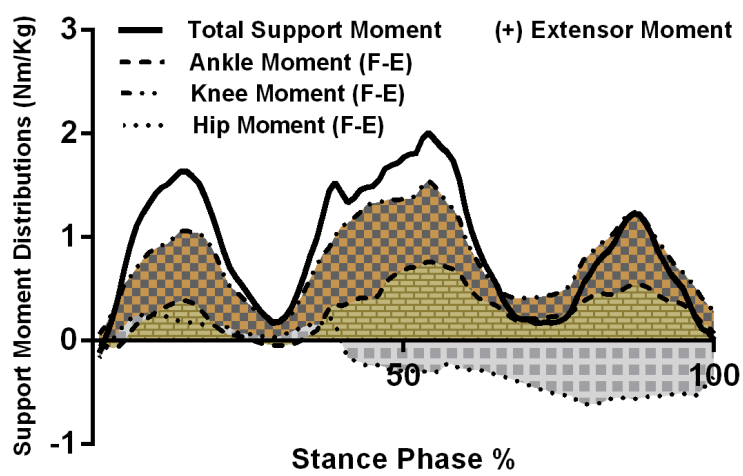

Figure 4: Illustration of time series of Individual joint distributions of total support moment during Tai Chi Gait (TCG). Extensor moment $(+)$.

\section{Tai Chi gait findings}

While performing TCG, subjects showed little variation in support strategy as shown in Figure 4. During TCG, knee involvement was greater in TCG (58.68 $\pm 1.84 \%)$. Following the knee, the ankle (32.78 \pm $1.25 \%)$ was the second most dominant contributor, and the hip (8.54 \pm 1.91\%) participated the least (Figure 3).

\section{Normal gait vs. Tai Chi gait}

Using a paired t-test, the contributions of each joint (ankle, knee, and hip) in NW and TCG were compared. In contrast to NW, TCG showed significantly different involvement of all three joints (Figure 5). The contributions of the knee (mean difference: $32.50 \pm 2.87 \%$ ) and hip (mean difference: $2.58 \pm 1.03 \%$ ) were found to be significantly greater in TCG than in NW $(\mathrm{p}<0.05)$. The ankle (mean difference: $35.08 \pm 2.87 \%)$ contributed less in TCG compared to NW $(\mathrm{p}<0.05)$.

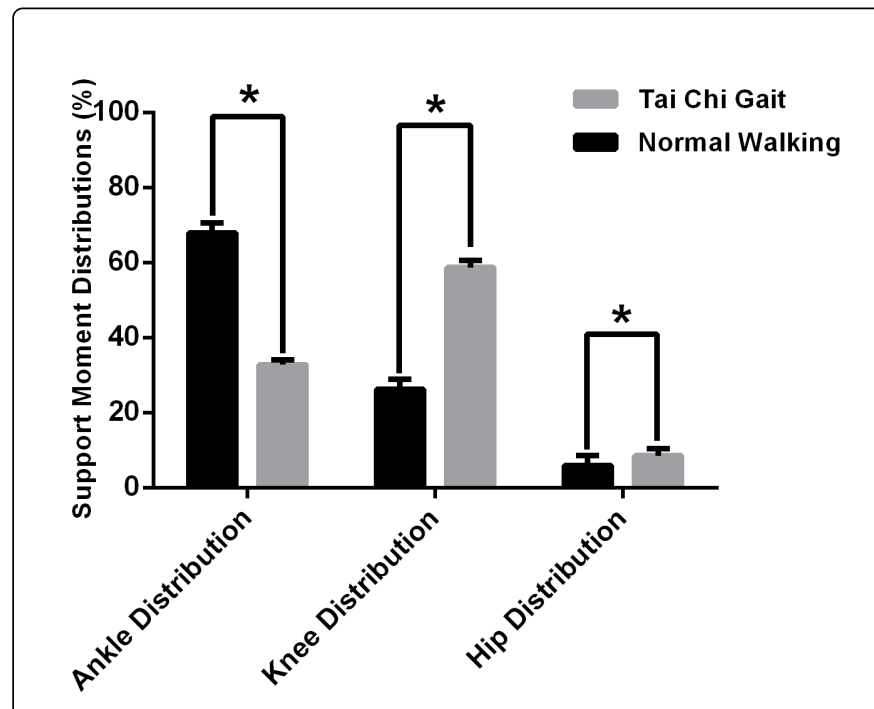

Figure 5: Comparison of the ankle, knee and hip joint moment distributions of total support moment between normal walking (NW) and Tai Chi gait (TCG). * Significant difference between NW and TCG $(\mathrm{P}<0.05)$.

\section{Discussion}

Our results for NW are consistent with those found in the literature. In our study, the ankle $(67.86 \pm 2.76 \%)$ was the primary contributor to the total support moment followed by the knee $(26.18 \pm 2.69 \%)$ and hip $(5.96 \pm 1.83 \%)$. Similar results were obtained by Torry et al. who determined the ankle, knee, and hip contributions $(71.73 \pm 14.90 \%$, $15.59 \pm 14.32 \%$, and $12.73 \pm 8.83 \%$, respectively) to the total support moment in a control group of healthy women [11]. DeVita and Hortobagyi also reported that the ankle is the dominant contributor to the total support moment [12].

Compared to normal walking, TCG was found to utilize a significantly different support pattern: the knee showed greatly increased contribution to the total support moment. Although there are no known studies of TCG using the total support moment, other studies have compared TCG to normal walking and inferences can be made from these findings. 
Several studies have noted the increased knee flexion required to perform TCG in comparison to NW. Wu reported an increase in duration and magnitude of knee and hip flexion by $139 \%$ and $66 \%$ respectively in elderly subjects performing TCG compared to NW [13]. In a similar study comparing TCG and NW, knee flexion in normal walking $\left(7.4^{\circ}-46.0^{\circ}\right)$ was observed to be less than in TCG $\left(13.1^{\circ}\right.$ $46.5^{\circ}$ ). During TCG, the knee was observed to be constantly flexed as compared to NW, which showed variable flexion over the gait cycle [14]. Taking into consideration that TCG requires constant knee flexion and is thus mechanically different in nature compared to NW, it is reasonable to see changes in the composition of the total support moment. As indicated by this study, a greater contribution of the knee is required to support the leg in the flexed stance that is necessitated by TCG. This pattern is further supported by electromyographic data of the various muscles of the leg.

Electromyographic evidence further corroborates the increased support moment contribution of the knee during TCG. The increased flexion of the knee in TCG has been shown to be correlated with increased activation of the knee extensors [14]. In one study, both the rectus femoris and tensor fasciae latae showed markedly increased activation magnitudes ( $403 \%$ and $252 \%$, respectively) in comparison to normal gait [13]. Greater peak muscle activity during TCG has also been observed in other knee extensor muscles, such as the vastus lateralis and vastus medialis [14]. The results of these electromyographic studies are further supported by observations of increased knee extensor strength in experienced Tai Chi practitioners [15].

While performing TCG, increased flexion of the knee and use of the knee extensors results in greater shear forces on the knee. Between the ankle, knee, and hip joints, the knee experiences the greatest peak shear force. Increased shear force during TCG is due to the greater knee flexion and displacement of the knee from the center of gravity. Although the peak shear force is increased in the knee during TCG, compressive force is reduced [9]. These increased forces on the knee further support our results, which indicate that the knee is the primary contributor to the total support moment during TCG. Because the contribution of the knee to the total vertical support is markedly increased when performing TCG, a strikingly high mechanical demand is placed on the knee joint.

Considering the load experienced by the knee, it is not surprising that research has shown Tai Chi to be beneficial to knee health. Both greater knee strength and proprioception have been documented. In Tai Chi intervention studies using healthy, elderly subjects, increased knee extensor strength in comparison to controls has been reported [15-17]. In addition to increased knee strength, Tai Chi training has been associated with improved proprioception about the knee compared to controls [17]. Several studies have examined the biomechanics of Tai Chi in healthy adults and have provided insight into the biomechanical mechanisms underlying the changes seen in practitioners of Tai Chi $[7-9,13]$.

Surprisingly, all biomechanical Tai Chi research to date has focused on healthy subjects. Although understanding the biomechanics of Tai Chi in people free of injury or disease is an important first step towards the evolution of Tai Chi as a therapy, if it is to be used for therapeutic purposes, the biomechanics of Tai Chi in subjects with disease must be elucidated. It is unclear how these people would handle the increased mechanical demand placed upon the knee during Tai Chi. For example, how would a person with knee osteoarthritis (OA) handle the increased knee mechanical stress associated with Tai Chi? Interestingly, there is evidence that Tai Chi is an effective therapy for people with knee OA. Interventional studies have shown that Tai Chi can reduce knee pain and stiffness as well as improve physical function $[4,18]$. These results are quite perplexing. Based on the current research, it would seem that the increased mechanical demand on the knee during Tai Chi would prove to be detrimental in people with knee OA. However, randomized controlled trials have provided evidence contrary to this conclusion. Future research into the biomechanics of Tai Chi in people with joint disease is required to answer these puzzling questions.

\section{Conclusions}

This study demonstrates different kinetics synergy patterns between Tai Chi gait and normal gait. Importantly, Tai Chi gait places a high mechanical demand on the knee joint. Our results provide biomechanical basis of Tai Chi's benefits on increasing knee joint range of motion and muscle strength, also suggest that prescribing Tai Chi as a potential therapy for people with joint disease, it needs a careful consideration of evaluating mechanical response of people with joint disease during Tai Chi exercise due to higher mechanical demand on the knee joint.

\section{Competing Interests}

None declared.

\section{Acknowledgements}

This study was supported by University Faculty Development Council Research Grant (W. Liu, PI). We thank Deana Rumble for her assistance on data collection.

\section{References}

1. Nahin RL, Barnes PM, Stussman BJ, Bloom B (2009) Costs of complementary and alternative medicine (CAM) and frequency of visits to CAM practitioners: United States, 2007. Natl Health Stat Report 18: 1-14.

2. Li F, Harmer P, Fitzgerald K, Eckstrom E, Stock R, et al. (2012) Tai Chi and postural stability in patients with Parkinson's disease. N Engl J Med 366: 511-519.

3. Li JX, Xu DQ, Hong Y (2009) Changes in muscle strength, endurance, and reaction of the lower extremities with Tai Chi intervention. J Biomech 42: 967-971.

4. Wang C, Schmid CH, Hibberd PL, Kalish R, Roubenoff R, et al. (2009) Tai Chi is effective in treating knee osteoarthritis: A randomized controlled trial. Arthritis Rheum 61: 1545-1553.

5. Jimenez PJ, Melendez A, Albers U (2012) Psychological effects of Tai Chi Chuan. Arch Gerontol and Geriatr 55: 460-467.

6. Jahnke R, Larkey L, Rogers C, Etnier J, Lin F (2010) A comprehensive review of health benefits of Qigong and Tai Chi. Am J Health Promot 24: e1-e25.

7. Chan SP, Luk TC, Hong Y (2003) Kinematic and electromyographic analysis of the push movement in Tai Chi. Br J Sports Med 37: 339-344.

8. Wu G, Liu W, Hitt J, Millon D (2004) Spatial, temporal and muscle action patterns of Tai Chi gait. J Electromyogr Kines 14: 343-354.

9. Wu G, Millon D (2008) Joint kinetics during Tai Chi gait and normal walking gait in young and elderly Tai Chi Chuan practitioners. Clin Biomech 23: 787-795.

10. Winter DA (2009) Biomechanics and Motor Control of Human Movement. 4th edition, John Wiley \& Sons, Inc. Hoboken, New Jersey. 
Citation: Pearlman R, Kovaleski JE, Wolfe J, Liu W (2017) The Joint Moment Distribution of the Lower Extremity During Tai Chi Gait. Altern Integr Med 6: 228. doi:10.4172/2327-5162.1000228

Page 5 of 5

11. Torry MR, Decker MJ, Ellis HB, Shelburne KB, Sterett WI, et al. (2004) Mechanisms of compensating for anterior cruciate ligament deficiency during gait. Med Sci Sport Exer 36: 1403-1412.

12. DeVita P, Hortobagyi T (2000) Age causes a redistribution of joint torques and powers during gait. J Appl Physiol 88: 1804-1811.

13. Wu G (2008) Age-related differences in Tai Chi Gait kinematics and leg muscle electromyography: A pilot study. Arch Phys Med Rehabil 89: 351-357.

14. Tseng SC, Liu W, Finley M, McQuade K (2007) Muscle activation profiles about the knee during Tai-Chi stepping movement compared to the normal gait step. J Electromyogr and Kines 17: 372-380.

15. Wu G, Zhao F, Zhou XL, Wei L (2002) Improvement of isokinetic knee extensor strength and reduction of postural sway in the elderly from long-term Tai Chi exercise. Arch Phys Med Rehab 83: 1364-1369.
16. Tsang WWN, Hui-Chan CWY (2005) Comparison of muscle torque, balance, and confidence in older Tai Chi and healthy adults. Med Sci Sport Exer 37: 280-289.

17. Li JX, Xu DQ, Hong Y (2008) Effects of 16-week Tai Chi intervention on postural stability and proprioception of knee and ankle in older people. Age Ageing 37: 575-578.

18. Brismee JM, Paige RL, Chyu MC, Boatright JD, Hagar JM, et al. (2007) Group and home-based tai chi in elderly subjects with knee osteoarthritis: a randomized controlled trial. Clin Rehabil 21: 99-111. 